\title{
DETECÇÃO DE TENDÊNCIAS MONÓTONAS EM SÉRIES MENSAIS DE PRECIPITAÇÃO PLUVIAL DO ESTADO DE SÃO PAULO ${ }^{(1)}$
}

\author{
GABRIEL CONSTANTINO BLAIN ${ }^{(2)}$
}

\begin{abstract}
RESUMO
O objetivo do trabalho foi detectar tendências climáticas nas séries de precipitação pluvial (PRE) de oito localidades do Estado de São Paulo, utilizando o método não paramétrico denominado teste sazonal de MannKendall (SMK). Quando aplicado à localidade de Monte Alegre do Sul, o SMK indicou a presença de tendências de elevação nas séries mensais de setembro e maio. Para as estações meteorológicas de Campinas e Ribeirão Preto, este método não paramétrico indicou a existência de tendências de elevação nas séries relativas ao mês de maio. Na série de Jundiaí foi verificada tendência de elevação apenas quando os totais anuais foram analisados. Nas localidades de Cordeirópolis e Pindorama, nenhuma tendência significativa foi observada. Na localidade de Ubatuba, foi detectada, em agosto, tendência de queda nos valores de PRE.
\end{abstract}

Palavras-chave: mudanças climáticas, teste de Mann Kendall.

\author{
ABSTRACT \\ DETECTING MONOTONOUS TRENDS IN MONTHLY RAINFALL SERIES OF THE \\ STATE OF SÃO PAULO, BRAZIL
}

The aim of the work was to evaluate climate trends in eight monthly rainfall series of the State of São Paulo, Brazil by using the non-parametric model called Seasonal Mann-Kendall test (SMK). Results of the SMK applied at the location of Monte Alegre do Sul shown the presence of increasing trends within the monthly series of May and September. At the weather stations of Campinas and Ribeirão Preto the results of this non parametric test indicated the presence of increasing trends within the monthly series of May. At the weather station of Jundiaí, the SMK indicated the presence of increasing trends only when annual precipitation amounts were evaluated. At the locations of Cordeirópolis and Pindorama, no significant trend was detected. At the weather station of Ubatuba, the SMK indicated the presence of decreasing trend within the monthly series of August.

Key words: climate change, seasonal Mann Kendall test.

(1) Recebido para publicação em 9 de abril de 2009 e aceito em 30 de março de 2010.

() Instituto Agronômico (IAC), Caixa Postal 28, 13001-970 Campinas (SP). E-mail: gabriel@iac.sp.gov.br (*) Autor correspondente. 
Segundo a nota técnica n. ${ }^{\circ} 79$ (Омм, 1966), o termo tendência climática é caracterizado por elevação ou diminuição suave e monótona nos valores de uma série temporal meteorológica. Neste contexto, observase que uma função, entre dois conjuntos ordenados, é denominada monótona quando há preservação ou inversão da relação de ordem entre os elementos desses dois agrupamentos. Dessa forma, métodos estatísticos que analisem a relação de ordem entre o tempo cronológico e os valores de uma variável meteorológica, caracterizando uma série temporal, podem fornecer informações importantes para a investigação de tendências de ordem climáticas.

De acordo com ВАск (2001), a identificação de alterações nos registros meteorológicos é de grande importância para os estudos de engenharia que utilizam as séries históricas, pois tanto as simulações como as aplicações de teorias de probabilidade são realizadas com a hipótese de que as séries históricas não permitem tendências. Ainda, segundo esse auto,r a dificuldade no estabelecimento de tendências significativas é devido à grande variabilidade natural dos dados meteorológicos.

Nesse aspecto, BLAIN (2009) indica que a principal característica dos dados mensais de precipitação pluvial (PRE) no Estado de São Paulo é sua elevada variabilidade temporal. Segundo esse autor, nos meses de verão, tais como janeiro e dezembro, podem ser observados regimes bastante elevados de PRE com medianas mensais próximas a $230 \mathrm{~mm}$; nos meses de inverno junho, julho e agosto - o regime desse elemento tornase similar ao de regiões semiáridas, com distribuição temporal fortemente assimétrica, quando há elevada probabilidade de ocorrência do valor nulo de PRE.

Considerando que trabalhos como os de HAYLOCK et al. (2006) indicam alterações no regime de precipitação pluvial na Região Centro/Sul do Brasil, o objetivo do trabalho foi detectar tendências monótonas no regime de PRE em oito localidades do Estado de São Paulo.

Foram utilizados dados mensais de precipitação pluvial disponibilizados pela Secretaria de Agricultura e Abastecimento do Estado de São Paulo (SAA/SP). Na figura 1 são ilustradas as coordenadas geográficas dos postos utilizados. À exceção da localidade de Pindorama (1951 a 2007), todas as demais localidades foram escolhidas a fim de compreender o período comum de 1948 a 2007.

Inicialmente, proposto por MANN (1945) e KENDALL e Stuart (1967), o teste não paramétrico de MannKendall (MK) pode ser definido, para uma série $Z=\{Z 1$, $\mathrm{Z} 2, \ldots \mathrm{Zi}, \mathrm{Zj}, \ldots . . \mathrm{Zn})$ por:

$$
\mathrm{T}=\sum_{\mathrm{j}<\mathrm{i}} \operatorname{sinal}\left(Z_{\mathrm{i}}-Z_{\mathrm{j}}\right) \text { em que: }
$$

$\operatorname{sinal}\left(Z_{i}-Z_{j}\right)=\left\{\begin{array}{l}1 ; \text { para } Z_{i}-Z_{j}>0 \\ 0 ; \text { para } Z_{i}-Z_{j}=0 \\ -1 ; \text { para } Z_{i}-Z_{j}<0\end{array}\right\}$

$$
\begin{aligned}
& \text { MK }=\left\{\begin{array}{l}
\frac{\mathrm{T}-1}{\sqrt{\operatorname{Var}(\mathrm{T})}} ; \text { para } \mathrm{T}>0 \\
0 ; \text { para } \mathrm{T}=0 \\
\frac{\mathrm{T}+1}{\sqrt{\operatorname{Var}(\mathrm{T})}} ; \text { para } \mathrm{T}<0
\end{array}\right\} \text { em que: } \\
& \operatorname{Var}(T)=\frac{n(n-1)(2 n+5)}{18}
\end{aligned}
$$

Observando as equações 1 e 2, verifica-se que o MK, quando comparado com outros métodos paramétricos é bastante robusto quanto a desvios da normalidade e não estacionalidade dos valores da série; justificando o fato do MK ser um teste muito utilizado em estudos de tendências em séries temporais. Contudo, é necessário ressaltar que a presença de sazonalidades ou autocorrelações significativas afeta a sensibilidade desse método (HAMED e RAO, 1998 e BAYAZIT e ONOZ, 2007). Observando a equação 2, tornase claro que a presença desses dois fatores aumenta a probabilidade de detecção de falsas tendências de elevação. Adotando-se como hipótese de nulidade (Ho), a inexistência de tendências climáticas e a presença de autocorrelação positiva aumentam a probabilidade do erro tipo I. Características opostas são observadas na presença de autocorrelação negativa.

A fim de adaptar o MK para séries autocorrelacionadas, bem como lidar com dificuldades impostas por possíveis sazonalidades, $\mathrm{HIRSCH}$ et al. (1982) e HiRsCH e SLACK (1984) propuseram um método denominado de Mann-Kendall Sazonal (SMK). No caso de séries mensais, o SMK deve ser calculado da seguinte forma: organizar os dados mensais em forma de matriz, em que as colunas são preenchidas com os valores da série relativos a cada mês do ano. Para o caso de, por exemplo, 50 anos de dados mensais, temse uma matriz (50x12; com os valores dentro de cada coluna classificados de acordo com o ano em ordem crescente). $\mathrm{O}$ fator $\mathrm{T}$ é inicialmente estimado para cada coluna (mês):

$$
\begin{aligned}
& \mathrm{S}=\sum_{\mathrm{j}=1}^{12} \mathrm{~T}_{\mathrm{j}} ; \text { para } \mathrm{T}_{\mathrm{j}}=\sum_{\mathrm{k}<1} \operatorname{sinal}\left(\mathrm{Z}_{\mathrm{lj}}-\mathrm{Z}_{\mathrm{kj}}\right) \\
& \text { no caso,j }=1 \ldots 12
\end{aligned}
$$




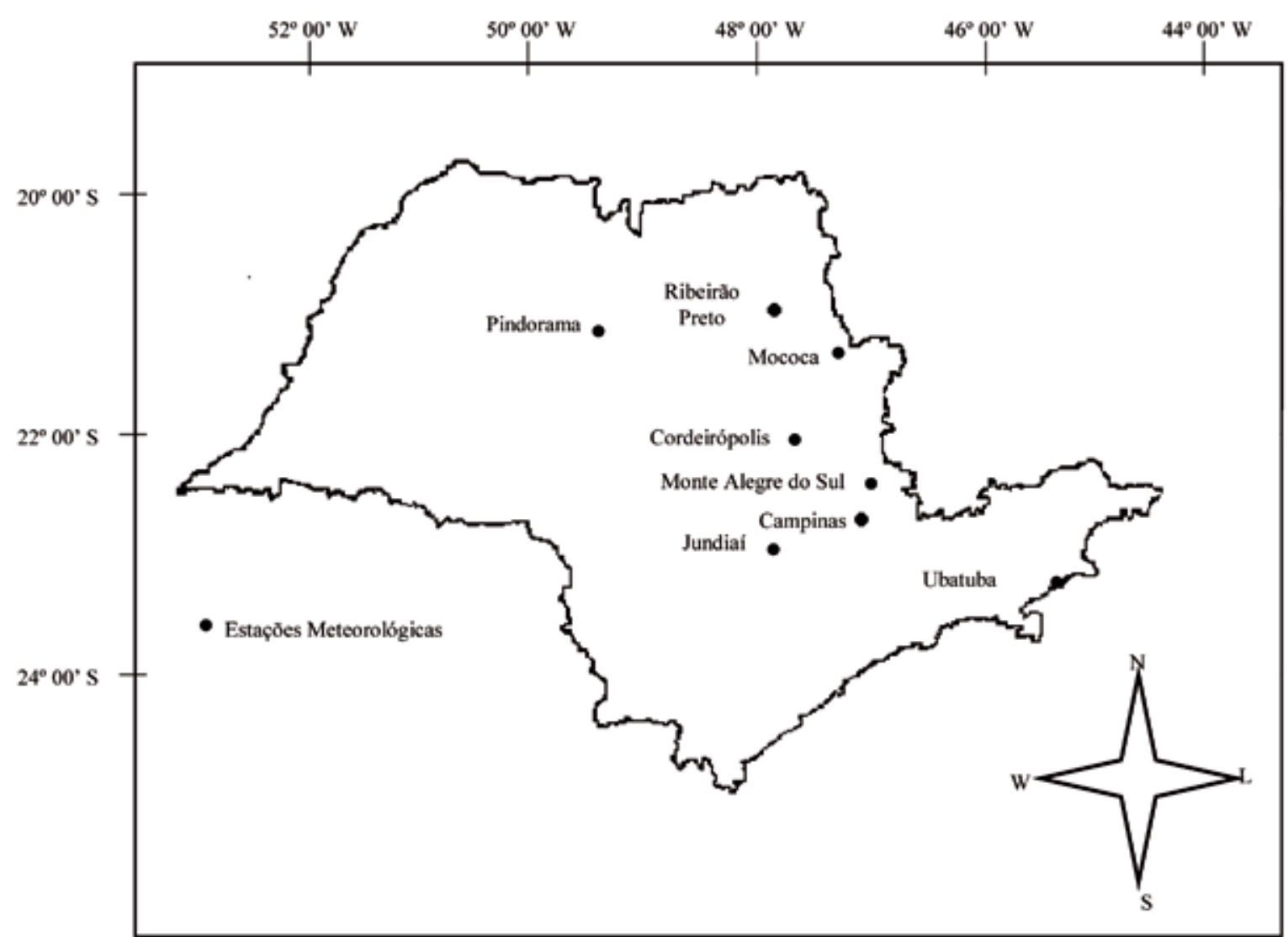

Figura 1. Postos meteorológicos pertencentes à Secretaria de Agricultura e Abastecimento do Estado de São Paulo.

$\operatorname{Var}(S)=\sum_{j=1}^{12} \operatorname{Var}\left(T_{j}\right)+\sum_{\substack{j, g=1 \\ g \neq j}}^{12} \operatorname{Cov}(T j: T g)$

$\operatorname{Var}\left(T_{j}\right)=\frac{n_{j}\left(n_{j}-1\right)\left(2 n_{j}+5\right)-\sum_{i=1}^{m} t_{\mathrm{i}}\left(t_{i}-1\right)\left(2 t_{i}+5\right)}{18}$

Em que $n_{j}$ é o número de valores de cada série $\mathrm{j}$ mensal (50 para a matriz 50x12), m é o número de grupos de valores adjacentes iguais e $t_{i}$ é o número de valores presentes em cada um desses grupos. A covariância entre duas estatísticas $T j T g$ é definida por:

$\operatorname{Cov}(\operatorname{TjTg})=\left\{\frac{S_{j g}+4 \sum_{m=1}^{n} \mathrm{R}_{m j} R_{m g}-n\left(n_{j}+1\right)\left(n_{g}+1\right)}{3} \mid\right.$

Em que são o número de valores presentes em cada série mensal, e:

$$
S_{j g}=\sum_{m<n} \operatorname{sinal}\left[\left(\mathrm{Z}_{n j}-\mathrm{Z}_{m j}\right)\left(\mathrm{Z}_{n g}-\mathrm{Z}_{m g}\right)\right]
$$

O fator R é a matriz ao rank (organização dos valores de cada mês em ordem crescente). O rank do m-ésimo elemento no j-ésimo mês é:

$$
\begin{aligned}
& R_{m j}=\left\{\frac{\mid n_{j}+1+\sum_{k=1}^{n} \operatorname{sinal}\left(x_{m j}-x_{k j}\right)}{2}\right\} \\
& \mathrm{SMK}=\left\{\begin{array}{l}
\frac{\mathrm{S}-1}{\sqrt{\operatorname{Var}(\mathrm{S})}} ; \text { para } \mathrm{S}>0 \\
0 ; \text { para } \mathrm{S}=0 \\
\frac{\mathrm{S}+1}{\sqrt{\operatorname{Var}(\mathrm{S})}} ; \text { para } \mathrm{S}<0
\end{array}\right\}
\end{aligned}
$$

Ho não é rejeitada se, em um teste bilateral, o valor absoluto de SMK for menor que um determinado valor $\mathrm{Z}$ da tabela da distribuição normal reduzida. Autores como MARENGO et al. (2007) utilizam o nível de significância $\alpha=0,05$. Adotando-se esse mesmo nível, Ho não será rejeitada sempre que $-1,96<\mathrm{SMK}<1,96$. Tendências de elevação ou queda resultam em valores positivos ou negativos de SMK. Verifica-se também que 
o SMK é, por definição, desenvolvido para detecção de tendências monótonas ou mudanças abruptas, uma vez quea relação de ordem entre os valores da série determina o valor final das equações 1 e 3 e, consequentemente, do próprio teste.

Observando as equações 3 e 9, nota-se que a introdução do fator $\sum_{\substack{j, g=1 \\ g \neq j}}^{12} \operatorname{Cov}(\mathrm{Tj}: \mathrm{Tg})$ eleva o valor final da equação 4, reduzindo esse valor da estatística SMK. Há, para casos de autocorrelação positiva entre os valores mensais, redução na probabilidade do erro tipo I. Utilizando dados hidrológicos, LETTENMAIER et al. (1994) afirma que a vantagem da aplicação do SMK está relacionada ao fato de que alterações em estações em que são esperados baixos valores das variáveis sob investigação não são influenciadas (dominated) por elevados valores oriundos de outras épocas.

A fim de facilitar a compreensão do teste SMK é proposto um exemplo hipotético de sua aplicação, ou seja: série de 60 anos de dados $(\mathrm{i}=1 . .60)$ compostas por três agrupamentos sazonais (S1 e S2). O agrupamento S1 é inteiramente aleatório. S2 é composta por dados aleatórios até os 30 anos iniciais. $\mathrm{O}$ valor relativo ao ano $31\left(\mathrm{~S}_{31}\right)$ é estimado como o maior valor ocorrido nos primeiros 30 anos dessa "estação". Os últimos 29 anos são estimados por $\left(\mathrm{S}_{\mathrm{i}}=\mathrm{S} 2_{\mathrm{i}-1}+0,05\right.$; para $\left.\mathrm{i}>31\right)$. $\mathrm{O}$ resultado do SMK é ilustrado na figura 2.

Utilizando o SMK foi possível detectar tendências presentes na série hipotética, bem como o agrupamento sazonal em que tal alteração foi observada. O SMK também indica que a alteração em $S 2$ foi de tal magnitude $(\mathrm{SMK}=7,35)$ que alterou de forma significativa a média anual dos valores da série $(\mathrm{SMK}=3,40)$.

Nas séries mensais em que foram observados valores significativos do SMK, realizou-se a análise de regressão linear simples, a fim de ilustrar a variabilidade da mesma. De acordo com autores como Sen (1968), Wang e SwaIl (2001) e HaYLock et al. (2006), o coeficiente angular (slope) dessa regressão, deve ser preferencialmente estimado com base no método de correlação de Kendall (Kendall's rank correlation), uma vez que o método dos mínimos quadrados é bastante sensível a valores extremos e à não-normalidade dos dados. Dessa forma, os coeficientes das regressões foram estimados conforme descrito em WANG e SWAIL (2000).

É importante ressaltar que, na literatura, é comum que dificuldades impostas por possíveis sazonalidades sejam contornadas por meio da subtração entre cada valor Zt e, no caso de séries meteorológicas mensais, as respectivas médias ou medianas de cada agrupamento mensal. Essa operação é usualmente denominada remoção da climatologia regional e seu resultado chamado anomalia ou resíduo mensal. O estudo de Blain (2009) é a aplicação do MK (com base apenas nas equações 1 e 2 ) em séries de resíduos mensais de precipitação pluvial no Estado de São Paulo, afirmando que a estimação desses resíduos deve ser feita por meio da subtração das medianas mensais, e não da média aritmética, tendo em vista a elevada variação temporal das formas das distribuições mensais de PRE no Estado de São Paulo. Em meses chuvosos, como janeiro ou dezembro, essas distribuições aproximam-se da forma gaussiana. Em meses com baixo regime de PRE, como julho ou agosto, essas distribuições chegam a assumir a forma exponencial ou " $\mathrm{j}$ " invertido.

Entretanto, BLAIN (2009) não considera que, mesmo utilizando o percentil fixo 0,5 , um determinado resíduo representa probabilidades de ocorrência bastante distintas considerando-se meses com diferentes curvas de probabilidade associadas aos totais de precipitação pluvial. Essa característica é exemplificada na tabela 1.

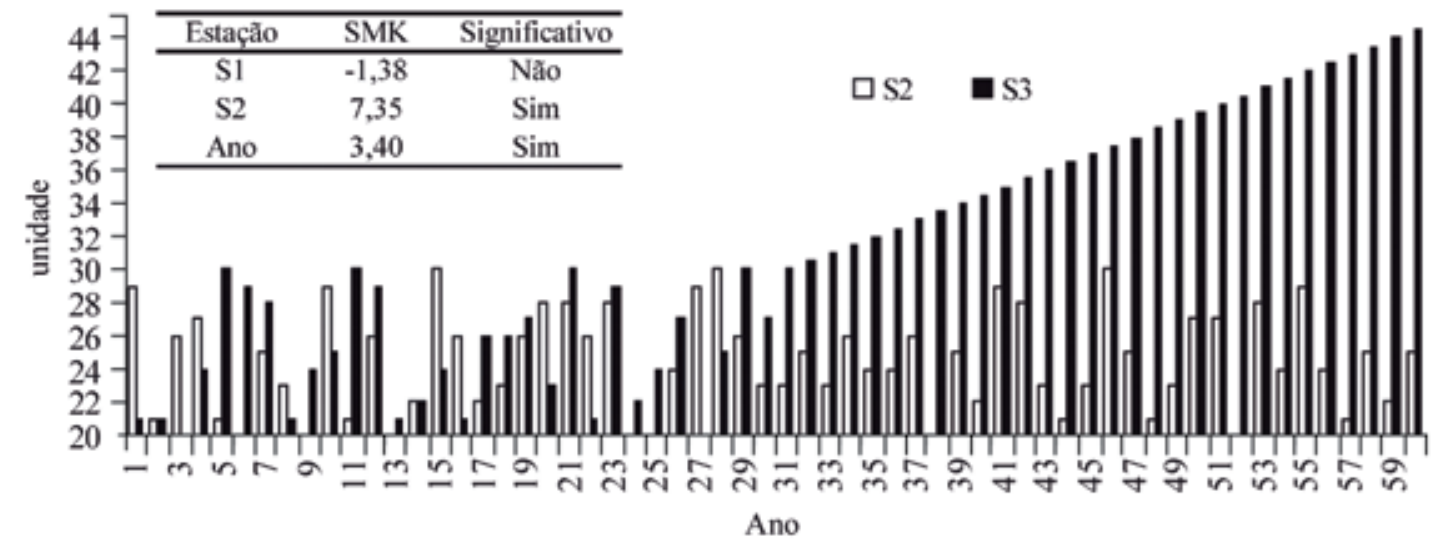

Figura 2. Exemplo hipotético do teste Mann-Kendall Sazonal (SMK) conduzido a 5\% de significância. 
$\mathrm{Na}$ tabela 1, verifica-se que o mesmo valor de resíduo $(30 \mathrm{~mm})$ pode indicar condições climáticas bastante distintas em diferentes meses no Estado de São Paulo. Dessa forma, as adaptações propostas por $\mathrm{HIRSCH}$ et al. (1982) e Hirsch e SLACK (1984), que resultaram no SMK, são as melhores alternativas para contornar as dificuldades impostas pela elevada variabilidade sazonal dos dados mensais de PRE nas investigações de tendências climáticas relativas a esse elemento meteorológico. Na tabela 2, são ilustrados os resultados da aplicação do SMK nas oito séries do estudo.

Considerando a escala anual, verifica-se na tabela 2 que, apenas nas localidades de Monte Alegre do Sul e Jundiaí, foram observadas tendências climáticas de elevação no regime de PRE. Por meio da análise mensal, nota-se que essa alteração está fundamentalmente relacionada aos meses de setembro e maio. Ressalta-se que as séries relativas a esse último agrupamento mensal possuem características monótonas e significativas de elevação nas localidades de Campinas e Ribeirão Preto.

O mês de agosto na localidade de Ubatuba foi o único com tendência significativa de redução no regime de PRE. Nesse aspecto, ainda que de forma pouco significativa, essa série litorânea revela, na escala anual, características qualitativas opostas, SMK negativo, às observadas nas demais localidades.

Nas figuras 3 e 4 são ilustradas as séries mensais com valores significativos do SMK. A fim de propiciar melhor visualização das tendências climáticas, as figuras 3 e 4 foram elaboradas utilizando-se o desvio ou diferença de cada valor de PRE mensal em relação à respectiva mediana amostral. Observando-se a figura 3 , verifica-se que o coeficiente angular, estimado pelos dois métodos propostos, corrobora o valor positivo do SMK descrito na tabela 2, indicando tendências de elevação no regime de precipitação pluvial nos meses sob investigação. Em contrapartida, na figura 4 a tendência de queda nos totais mensais de agosto na localidade de Ubatuba é bastante evidente. A análise das figuras 3 e 4 também indica que as tendências detectadas ocorreram de forma suave no tempo, isto é, não são oriundas de mudança abrupta ou salto climático.

Entre as oito localidades analisadas, nas séries de Monte Alegre do Sul e Jundiaí foram detectados indícios significativos de elevação no regime de precipitação pluvial, considerando a escala anual. Essa alteração climática está, na localidade de Monte Alegre do Sul, temporalmente localizada em maio e setembro.

No agrupamento mensal de maio, são observados valores positivos do SMK em todas as séries, e nas localidades de Campinas e Ribeirão Preto, esse teste indica tendências significativas de elevação nos valores mensais de precipitação pluvial.

Tabela 1. Diferentes probabilidades de ocorrência associadas a um mesmo valor de resíduo de precipitação pluvial em Campinas, quando são considerados diferentes meses

\begin{tabular}{lccc}
\hline Mês & Mediana & $\begin{array}{c}\text { Desvio da } \\
\text { mediana }\end{array}$ & $\begin{array}{c}\text { Probabilidade } \\
\text { acumulada }\end{array}$ \\
\cline { 2 - 3 } Janeiro & 229 & $\mathrm{~mm}$ & \\
Julho & 16 & 30 & $\begin{array}{c}(259 \mathrm{~mm}) 0,60^{*} \\
(46,2 \mathrm{~mm}) 0,79^{*} \\
\text { Mês }\end{array}$ \\
Janeiro & 248 & $\begin{array}{c}\text { Desvio da } \\
\text { média }\end{array}$ & $\begin{array}{c}\text { Probabilidade } \\
\text { acumulada }\end{array}$ \\
Julho & 30 & 30 & $(278 \mathrm{~mm}) 0,66^{*}$ \\
\end{tabular}

* Estimadas por meio da distribuição gama conforme BlAIN (2009).

Tabela 2. Estatística SMK relativa ao teste Mann-Kendall Sazonal nas localidades de Campinas, Cordeirópolis, Jundiaí, Mococa, Monte Alegre do Sul, Pindorama (1951/2007), Ribeirão Preto e Ubatuba, pertencentes ao Estado de São Paulo. Nas sete localidades consideraram-se os anos de 1948 a 2007

\begin{tabular}{lcccccccc}
\hline Meses & Cps. & Cord. & Jund. & Moc & Mte. Alegre & Pind. & R.Preto & Ubatuba \\
\hline janeiro & 1,12 & 0,59 & 1,65 & 1,80 & 0,60 & 0,03 & 0,91 & $-1,01$ \\
fevereiro & $-0,59$ & $-0,98$ & $-0,81$ & $-0,50$ & $-0,89$ & 0,58 & 0,00 & $-1,29$ \\
março & $-0,03$ & $-0,14$ & 0,29 & $-1,34$ & $-0,15$ & 0,90 & $-0,92$ \\
abril & $-0,57$ & 0,93 & $-0,06$ & 0,69 & 0,63 & 0,21 & $-0,13$ & $-0,96$ \\
maio & $2,42^{*}$ & 1,00 & 1,81 & 1,76 & $2,04 *$ & 0,15 & $2,05 *$ & 0,77 \\
junho & $-0,68$ & $-0,39$ & $-0,57$ & $-1,47$ & $-0,61$ & 0,09 & $-0,81$ & 0,06 \\
julho & 0,99 & 1,07 & 1,66 & 1,34 & 0,98 & 0,09 & 0,99 & $-0,04$ \\
agosto & $-0,50$ & $-0,85$ & $-0,08$ & $-0,61$ & $-0,62$ & 0,32 & $-0,60$ & $-2,32^{*}$ \\
setembro & 1,28 & 1,12 & 1,75 & 1,49 & $2,18^{*}$ & 0,36 & 1,82 & 1,45 \\
outubro & $-0,55$ & $-1,12$ & $-0,75$ & $-0,33$ & 0,96 & 0,49 & $-1,45$ & 0,46 \\
novembro & 0,82 & 0,77 & 1,91 & 0,42 & 0,85 & 0,42 & 1,39 & 0,10 \\
dezembro & 0,77 & $-0,22$ & 0,82 & 0,69 & 0,90 & 0,99 & $-0,34$ & $-1,31$ \\
Ano & 1,24 & 0,47 & 1,97 & 1,00 & $1,97 *$ & 1,14 & 0,79 & $-0,75$ \\
\hline
\end{tabular}

\footnotetext{
* : Significativo a $5 \%$.
} 

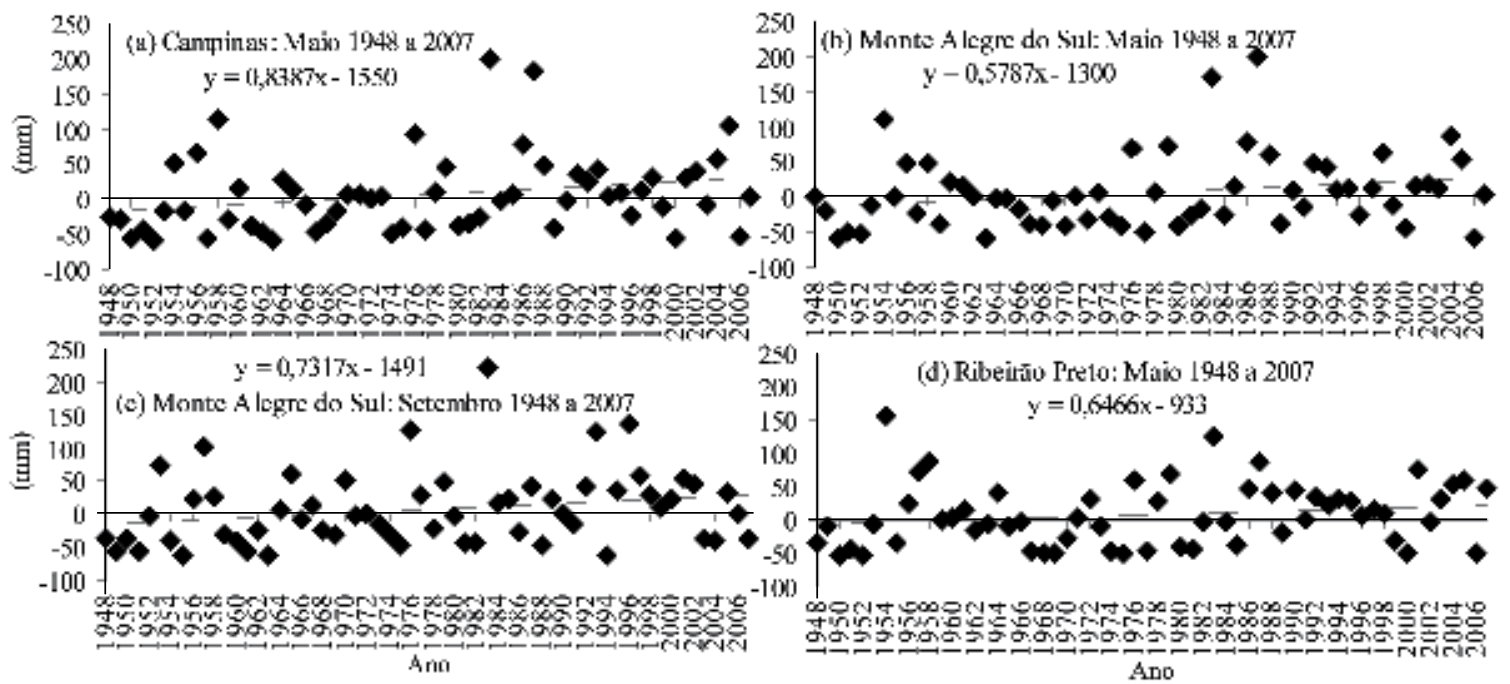

Figura 3. Análise de regressão linear aplicada às séries de precipitação pluvial com tendências de elevação significativas, a 5\% de significância e de acordo com o método de Mann-Kendall Sazonal. Os dados são resultantes da subtração entre cada valor mensal de precipitação pluvial e as respectivas medianas amostrais. Os valores dessa medida de posição central é, em milímetros, 59 (a); 61 (b); 60 (c) e; 51(d).

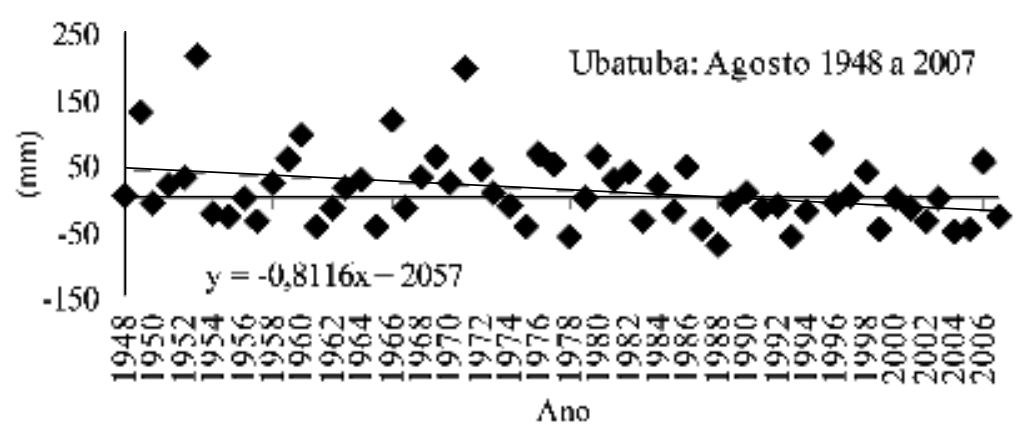

Figura 4. Análise de regressão linear aplicada às séries de precipitação pluvial da localidade de Ubatuba com tendências de elevação significativas, a 5\% de significância e de acordo com o método de Mann-Kendall Sazonal. Os dados são resultantes da subtração entre cada valor mensal de precipitação pluvial e a mediana amostral. O valor dessa medida de posição central é de 80 milímetros.

Embora com baixa significância, na localidade de Ubatuba, são observados indícios de queda, especialmente em agosto, nos valores de precipitação pluvial. Essa característica é oposta à observadas nas demais localidades. De forma geral, não há, considerando as oito localidades analisadas, marcantes indícios de tendências climáticas nos totais mensais de precipitação pluvial no Estado de São Paulo.

\section{REFERÊNCIAS}

BACK, A.J. Aplicação de análise estatística para identificação de tendências climáticas. Pesquisa Agropecuária Brasileira, v. 36, p.717-726, 2001.

BAYAZIT, M.; ONOZ, B. To prewhiten or not prewhiten in trend analysis? Hydrological Science Journal, v.52, p.611-624, 2007.
BLAIN, G., C., Considerações estatísticas relativas à oito séries de precipitação pluvial da secretaria de agricultura e abastecimento do Estado de São Paulo. Revista Brasileira de Meteorologia, v.24, p.12-23, 2009

HAMED, K.H.; RAO, A.R. A modified Mann-Kendall trend test for auto-correlated data. Journal of Hydrology, v.204, p.182-196, 1998.

HAYLOCK, M.R.; PETERSON, T.C.; ALVES, L.M.; AMBRIZZI, T.; ANUNCIAÇÃO, Y.M.T.; BAEZ, J.; BARROS, V.R.; BERLATO, M.A.; BIDEGAIN, M.; CORONEL, G.; CORRADI, V.; GARCIA, V.J.; GRIMM, A.M.; KAROLY, D.; MARENGO, J.A.; MARINO, M.B.; MONCUNILL, D.F.; NECHET, D.; QUINTANA, J.; REBELLO, E.; RUSTICUCCI, M.; SANTOS, J.L.; TREBEJO, I.; VINCENT L.A. Trends in total and extreme South American rainfall in 1960-2000 and links with sea surface temperature. Journal of Climate, v.19, p.1490-1512, 2006. 
HIRSCH, R.M.; SLACK , J.R. A nonparametric trend test for seasonal data with serial dependence. Water Resources Research, v.20, p.727-732, 1984.

HIRSCH, R.M.; SLACK J.R.; SMITH, R.A. Techniques of trend analysis for monthly water quality data. Water Resources Research, v.18, p.107-121, 1982.

KENDALL, M.A.; STUART, A. The advanced theory of statistics. Londres: Charles Griffin, 1967. 690p.

LETTENMAIER, D.P.; WOOD, E.F.; WALLIS, J.R. Hydroclimatological trends in the continental United States, 1948-88. Journal of Climate, v.7, p.586-607, 1994.

MANN, H.B. Non-parametric tests against trend. Econometrica, v.13, p.245-259, 1945.
MARENGO, J.; NOBRE, C.; RAIGOZA, D.; VALVERDE, M.; PISNITCHENKO, I.A.; OLIVEIRA, J.C.M. Boletim do Projeto: uso de cenários de mudanças climáticas regionais em estudos de vulnerabilidade e adaptação no Brasil e na América do Sul (GOF-UK-CPTEC), 2007. Disponível em: <www.cptec.inpe. br/mudancas_climaticas $>$. Acesso em: 1. ${ }^{\circ}$ dez. 2008.

ORGANIZAÇÃO METEOROLÓGICA MUNDIAL: Climatic Change. Geneva, Switzerland: OMM, 1966. 80p.

Sen, P.K., Estimates of the regression coefficient based on Kendall's tau. Journal of American Statistic Association. v.63, p.1379-1389, 1968

WANG, L.X., SWAIL, V.R., Changes of extreme wave heights in northerner hemisphere oceans and related atmospheric circulation regime. Journal of Climate. v.14, p. 2204-2221, 2001. 
